\title{
LOCAL IN-SITU ANALYSIS OF PEM FUEL CELLS BY IMPEDANCE SPECTROSCOPY AND RAMAN MEASUREMENTS
}

\author{
E. Gülzow ${ }^{\text {a) }}$, M. Schulze ${ }^{\text {a) }}$, K. A. Friedrich ${ }^{\text {a) }}$, P. Fischer ${ }^{\text {b) }}$ and H. Bettermann ${ }^{\text {b) }}$ \\ a) Institute of Technical Thermodynamics, German Aerospace Center (DLR), \\ Stuttgart, Germany \\ ${ }^{b)}$ Institute of Physical Chemistry I, Heinrich-Heine-Universität Düsseldorf \\ Düsseldorf, Germany
}

\begin{abstract}
An understanding of the processes inside of low temperature fuel cells on a local scale is required for an effective improvement strategy. For this purpose in situ Raman spectroscopy and local impedance spectroscopy is being developed. The contribution describes the modifications to the cell, and installations of additional devices and the experimental detection systems for integrating both methods into a single cell set up. First results to verify the combined results were carried out and are presented. In the case of the local impedance with segmented cells the additional effort for the simultaneous frequency analysis of all segments is described. The Raman signals of hydrogen, oxygen and water from the channels of the flow field are shown and first measurements of gas composition along the fuel path are analyzed.
\end{abstract}

\section{Introduction}

Physico-chemical processes inside fuel cells are quite complex involving many influencing parameters and despite extensive research activities in the past they are still not completely understood. An important aspect in this respect is that processes in fuel cells proceed on small scales but are mainly investigated by global methods. To take a closer look at those processes and to validate theoretical simulations (1), spatially resolved data are essential. In the recent years various physico-chemical methods have been applied to study fuel cells in operation that are primarily focused on the changes of electric parameters $(2,3)$.

A lot of different analytic methods can be used for the in-situ diagnostic of fuel cells. Current-voltage (V-I) characteristics and electrochemical impedance spectroscopy (EIS) are commonly used to characterize fuel cells, but these methods do not give locally resolved information if the cell is not segmented. For a correlation with simulation which can be evaluated on different scales locally resolved experimental information is required.

A commonly used experimental approach for local investigations is the measurement of current densities that are related to local electrochemical mass conversions (4). For measuring local current densities, a device based on a printed circuit board has been developed by the German Aerospace Center (DLR) (5) and licensed for commercial use. This printed board setup can easily be adapted to all kinds of flow field architectures.

Electrochemical impedance spectroscopy (EIS) is frequently used to investigate fuel cells. With EIS some of the relevant electrochemical fuel cell processes can be identified and associated to losses in the cell. EIS gives information about the transport processes and 
the electrochemical kinetics (6), but EIS has also some disadvantages - one is the rather long time required for recording an electrochemical impedance spectrum (depending on the lower frequency limit of the spectrum, several minutes up to one hour is needed). Furthermore, EIS is typically used as a global method and consequently yields no local information.

An alternative approach for achieving locally resolved data has been realized by applying Raman spectroscopy to the study of a single cell. Raman spectroscopy is an optical scattering method to detect molecular vibrations and rotations (the Raman effect was discovered by the Indian physicist Chandrasekhara Venkata Raman $(1888-1970)$ in 1928 who received the Nobel prize two years later). Raman spectroscopy allows investigating gas compositions and can be performed online in the cell without removing samples for external analysis. Consequently, Raman spectroscopy can be used as an minimal invasive method for gas analysis in fuel cells. Presently Raman spectroscopy is frequently used in the fuel cell research for the ex-situ characterization of fuel cell components like the polymer electrolyte membrane, but only few groups use the Raman spectroscopy as an in-situ method.

With regard to the in-situ investigations of fuel cell processes, Raman spectroscopy belongs to the few methods which are able to analyze homo-nuclear diatomic gases or mixtures of them quantitatively. Furthermore, Raman signals are generally less interfered by the presence of liquid water.

The spectroscopic setup presented in this contribution is mainly concerned with the investigation of gas compositions within flow fields. Locally current density distribution and EIS can be achieved with a segmented cell set up. This information can be combined with the time evolution of gaseous species at different places along the serpentine flow path. For this a multi-fiber Raman spectroscopic setup was developed at the University of Düsseldorf. Supplementary to the mere measuring of changes within distribution of gases, the goal of this setup is to identify the mutual interdependencies among the reactant gases as well as water vapor and liquid water.

This contribution is divided into two parts. The first part is concerned with measurements of local current density whereas the second part outlines the Raman setup. The combination of both experimental setups will provide concise information about actual operating states and it is thought to use the combination of both experimental approaches for pursuing degradations as well as describing the processes inside fuel cells in greater detail.

\section{Experimental}

The technical setup of the locally resolved electrochemical impedance spectroscopy

At DLR various techniques have been developed for measuring current density in fuel cells (5). Currently a printed circuit board (PCB) is used as a tool for the current density measurements. The side of the board facing the electrode exhibits a normal machined flow field. Fig. 1 shows the standard laboratory cell of DLR with the printed circuit board for the current density measurement. The flow field on the printed board is segmented and the dimensions of the segments define the local resolution of the current density measurement. Each segment is connected via separate resistances to the back side of the 
PCB, which serves as the current collector (Fig. 2). The voltage drop, which correlates to the current in the related segment, is measured at each resistance. In addition, sensors integrated in the PCB enable the measurement of temperatures at different positions. The measuring time is limited by the measurement rate of the equipment used for the voltage measurements.

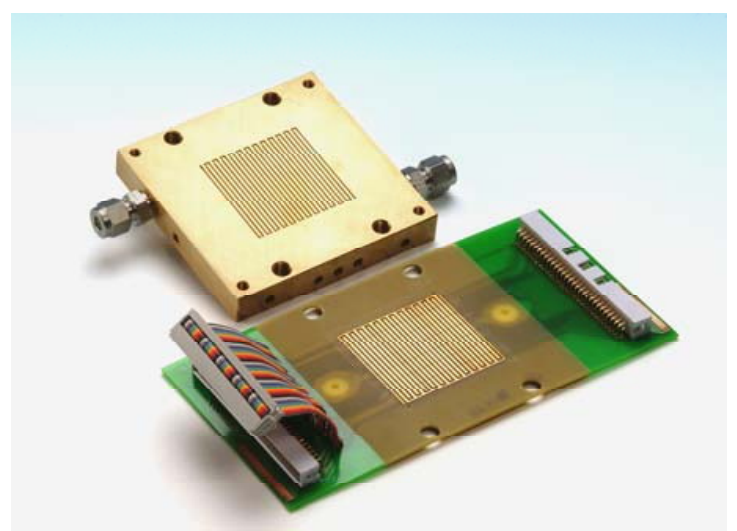

Fig. 1: Photography of the DLR laboratory cell with printed circuit board for current density measurements

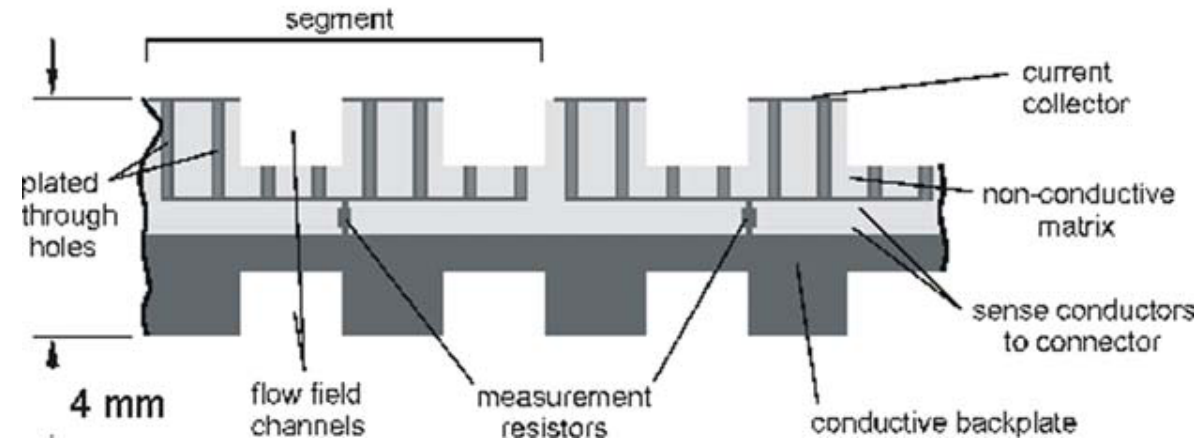

Fig. 2: Scheme of the printed circuit technology for current density measurements

The PCB technique for current density measurement is upgraded presently to integrate local EIS (Fig. 3), which provides information about the local membrane resistance, electrochemical reaction, and transport processes. The issues associated with the implementation of locally resolved impedance spectroscopy, as well as envisioned solutions are presented in this contribution.

Integration of locally resolved EIS is a difficult task because it results in long measurement times, especially if the different segments are measured sequentially. EIS is only valid if the system's response is linear and does not change during the measurement which, however, requires a stabilization time. Fuel cells show an instability when changing operation conditions which has to be considered in the measurement. Therefore, it is advantageous to measure the individual segments simultaneously. As a consequence of the PCB design the AC signal for the impedance spectroscopy is very small and difficult to detect. The measurement system developed at DLR to overcome these issues uses a multi-channel lock-in amplifier technique to enhance the small signals and to measure the segment simultaneously. 


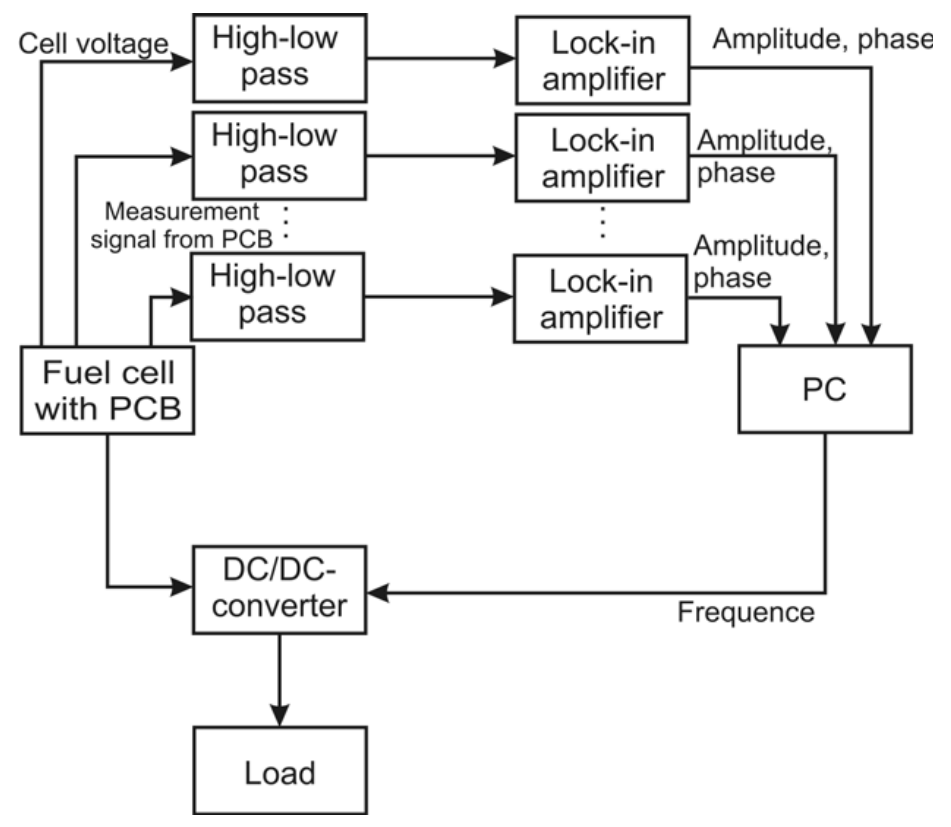

Fig. 3: Scheme of the system for locally resolved electrochemical impedance spectroscopy

An additional advantage of this system is that the excitation does not have to be sinusoidal, which allows a simply integration of the excitation generator into the DC/DCconverter. As another consequence of the flexibility regarding the excitation, this measurement technique can also be used for EIS measurements on individual cells in a stack, because the problem that the behaviour of the individual cells in the stack does not scale with the stack behaviour (non linear system) can be neglected. The multi-channel lock-in amplifier is realized by individual amplifiers and a digital signal processor (DSP) or few of digital signals processors depending on the numbers of channels, which are measured simultaneously.

With the DSP also the normal current density measurement can be improved. The main advantage is that the different channels (segments) will not measured sequentially but simultaneously - so the sample rate will be accelerated from presently 100 segments/s for a digital voltmeter to approx. 40000 images/s (complete current density distribution images).

\section{Raman multi-fiber setup}

The multi-fiber spectrometer consists mainly of an excitation laser, twin fiber units and a spectrograph-detector assembly (Fig. 4). Raman scattering is generated by a frequencydoubled thin disk Yb:YAG laser (515 nm, two beams/ up to $14 \mathrm{~W})$. Its beam is first homogenized and then coupled into the excitation fibers (multimode-step-index quartz hardcoat/low $\mathrm{OH}$ content). Along a single serpentine flow field seven observation ports are installed in the test fuel cell. The observation port consists of a small opening to the channel (diameter $1 \mathrm{~mm}$ ) that is sealed with a micro-lens assembly that is built up by two aspheric lenses. Each port is equipped with a set of twin fibers that is housed in a SMAconnector. The micro-lens assembly within the SMA-connector focuses the laser light 
into the flow field canal. The lenses also collect the back-scattered Raman emission.

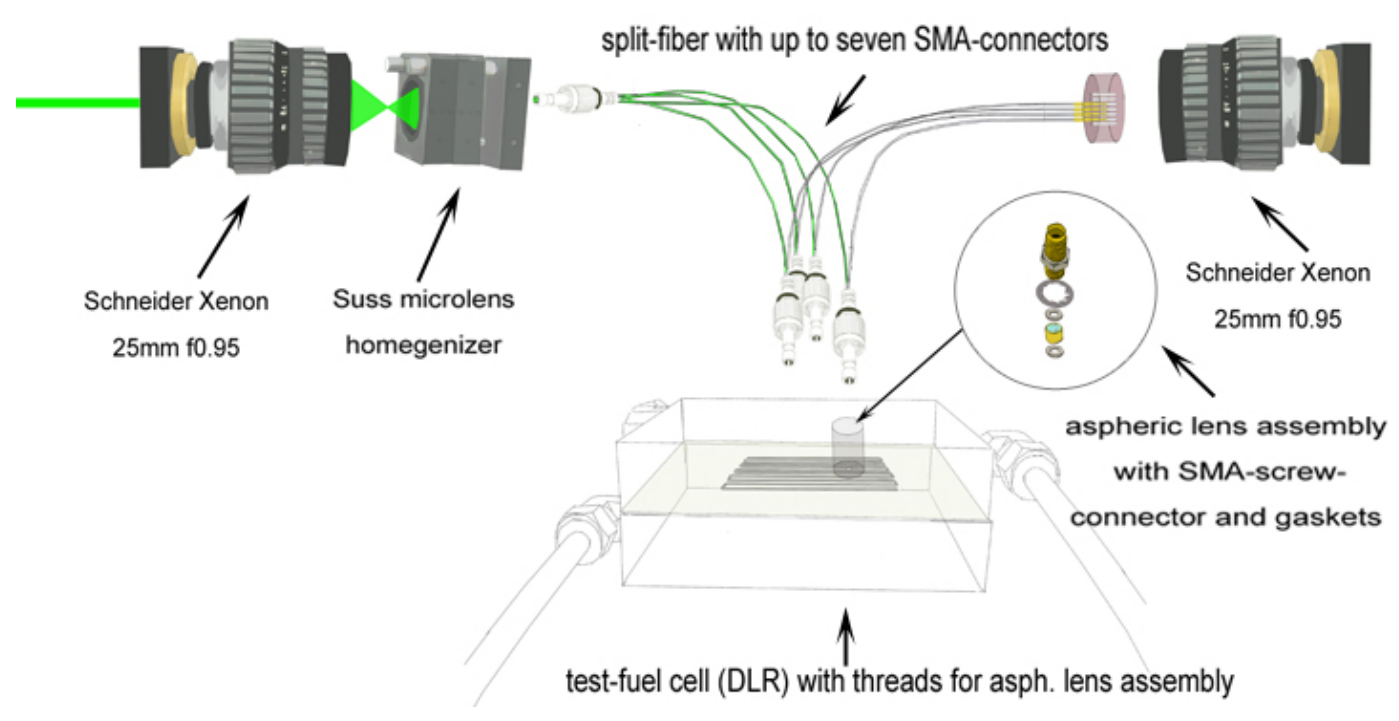

Fig. 4: Schematic drawing of multi-fiber Raman assembly

The second fiber that accompanies each excitation fiber directs the Raman light to a selfbuilt grating spectrograph of very high luminosity. The spectrograph contains a notch filter for rejecting Rayleigh scattering. The diffracted Raman light is then imaged with a liquid nitrogen cooled charged coupled device (CCD) camera. This setup permits the recording of spectra in a few minutes.

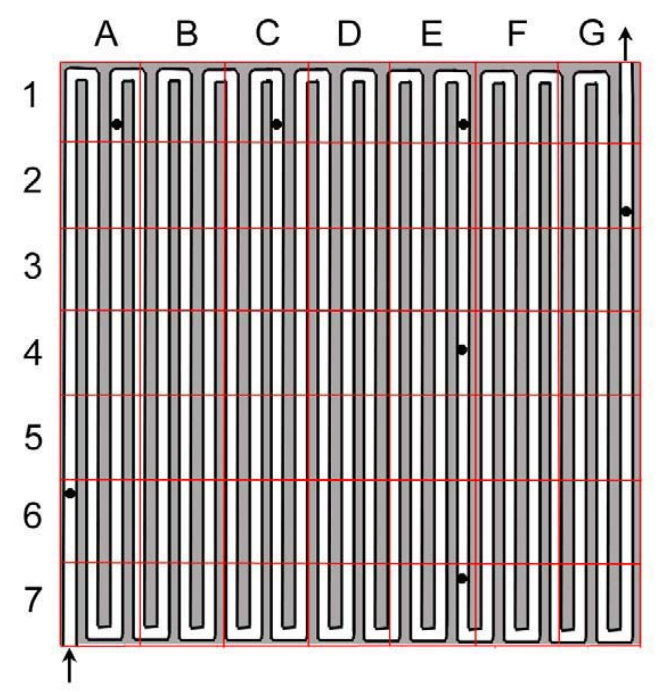

Fig. 5: Flow field of the test cell, dots mark the positions of the observation ports. The arrows mark the entrance and the exit of gas flow in a co-flow configuration. The segments from which local current densities are achieved are indicated by 1 to 7 and $A$ to $G$ 
The observation ports along the flow field serpentine are presented in Fig. 5. To observe the spatial distribution of the gaseous species along the serpentine, the ports are first located at the entrance and the exit of the fuel cell serpentine. The remaining ports record the gas distribution along a linear slope of the flow field ( 3 ports in a row) as well as before or after a U-turn. The ports correspond to special segments of the local current density measurements. After proving the detection sensitivity of gases within the flow field by Raman spectroscopy, it was verified that the chosen laser excitation powers does not influence the fuel cell processes significantly. For this, one bipolar plate was removed and substituted by a thermographic camera that monitored the temperature of the GDL from the back side.

The heating up of the GDL depends on the two possible aligned positions of the twin fibers (Fig. 6). In Fig. 6 a, the optical fibers are arranged with a parallel offset with regard to channel position. In this case the complete laser beam hits the GDL surface which is absorbimg and the laser spot on the GDL increases the temperature locally significantly. On the other hand, positioning the fiber aligned at the center on top of the channels (Fig. $6 \mathrm{~b})$, the diverging laser light is first reflected at the channel wall and then further diverged within the channel. This expansion of beam leads to a considerable reduction of power density at the surface of the GDL. Since the temperature increase only amounts 8.5 $\mathrm{K}$ for a $100 \mathrm{~mW}$ output power at the fiber exit, this fiber position has been used for the experiments.

\section{Fuel cell test facility}

The PEM test fuel cell used for Raman experiments and the locally resolved electrochemical impedance spectroscopy exhibits an active surface area of about $25 \mathrm{~cm}^{2}$. The MEA from Ion Power Inc. consists of a commercially available sulfonated perfluorinated membrane with equal platinum loading $\left(0.6 \mathrm{mg} \mathrm{cm}^{-2}\right)$ on both electrodes. The GDL is made from carbon paper. Both flow fields are single serpentine channels milled into stainless steel plates that are completely gold-plated.

The fuel cell is controlled by a test station developed by DLR (Fig. 8). The test station controls the humidification of the cell by maintaining a defined temperature of both the fuel cell and the humidifiers, as well as controlling gas flows and pressures on both the anode and cathode side. The gases are externally humidified by two separate humidifiers. The fuel cell is operated in a constant voltage mode. Co-flow configuration and a flow rate with a stoichiometric factor $\lambda$ of 1.5 for a current density of $1 \mathrm{~A} \mathrm{~cm}^{-2}$ is used. The cell temperature is $75^{\circ} \mathrm{C}$.
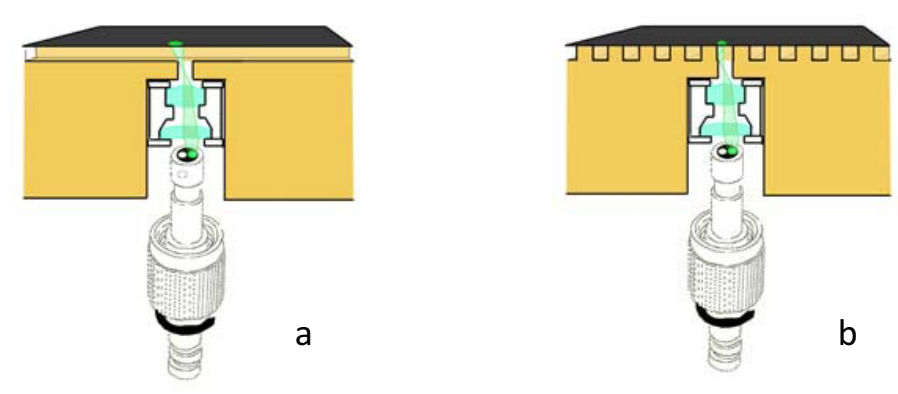

Fig. 6: The two twin fiber positions relative to the orientation of the flow field channels. 


\section{Results}

\section{Raman measurements of gaseous species}

Diatomic molecules possess only one vibration which stretches and compresses the bond. In the case of homo-nuclear molecules this vibration is detectable by Raman spectroscopy but not by infrared absorption. In the gaseous state in which molecules move more or less independently, vibrational motions are generally coupled with molecular rotations. With regard to quantum mechanic selection rules, Raman scattering of diatomic molecules is observed for rotational-vibrational transitions in which the rotational state specified by the rotational quantum number $J$ remains constant $(Q$ branches), is decreased (O branch) or is increased ( $\mathrm{S}$ branch) by two units (7). The most intense rotational-vibrational transition within the spectrum of molecular hydrogen (4156 $\mathrm{cm}^{-1}$ ) corresponds to the $\mathrm{Q}$ branch for which the initial and the final $\mathrm{J}$ is 1 (Fig. 7) (8).

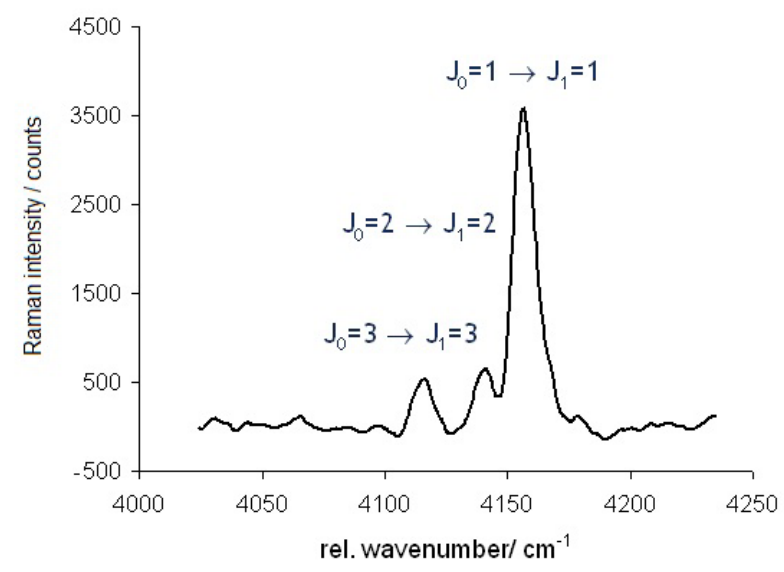

Fig. 7: Vibrational-rotational Raman spectrum of hydrogen $\left(4156 \mathrm{~cm}^{-1}\right)$ recorded from the anodic flow field. The transitions are assigned by the initial and final rotational quantum numbers. Laser output power $2 \mathrm{~W}, \lambda=477 \mathrm{~nm}$, time of measurement: $5 \mathrm{~min}$, cell temperature: $78^{\circ} \mathrm{C}$, temperature of the humidifier: $80^{\circ} \mathrm{C}$.

The prominent $\mathrm{Q}$ branch of the rotational-vibrational transition of oxygen is recorded at $1555 \mathrm{~cm}^{-1}$ (9) and that of nitrogen is detected at $2331 \mathrm{~cm}^{-1}$. Because of the different scattering cross sections, the Raman signal intensity of $\mathrm{O}_{2}$ is around one fourth of that of $\mathrm{H}_{2}$ (10). This means that studies of oxygen requires four times longer detection times or corresponding laser powers to achieve a similar quality of Raman signals as known from measuring hydrogen.

Beside the detection of molecular vibrations, Raman transitions also result from changes of pure rotational states (11). A part of the pure rotational spectrum of hydrogen is shown in Fig. 8. The signal intensities of rotational transitions depend on the temperature of the sample. Using a simple Boltzmann distribution the local temperature can be determined in the direct surroundings of the observation port. 


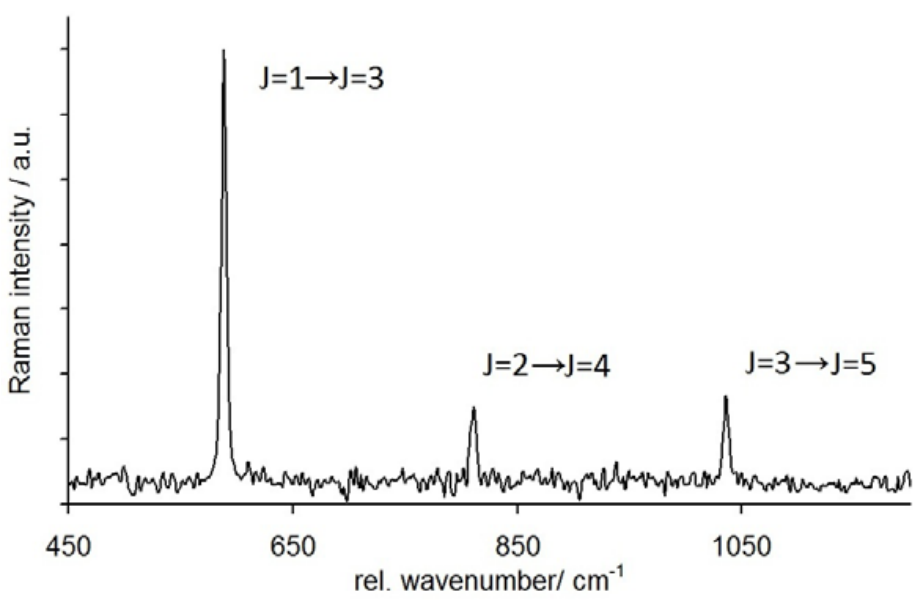

Fig. 8: Rotational Raman spectrum of hydrogen recorded from a flow field channel. Cell temperature $64^{\circ} \mathrm{C}$, Laser output power $2 \mathrm{~W}, \lambda=477 \mathrm{~nm}$, recording time $5 \mathrm{~min}$.

All three vibrations of water (a symmetric $\mathrm{OH}$ stretch mode, an asymmetric $\mathrm{OH}$ stretch mode and the angle-deformation mode) are Raman-active (12). In the gaseous state a sequence of rotational-vibrational transitions is generally detectable but measuring inside a flow field, a single Q branch of the symmetric stretch vibration $\left(3652 \mathrm{~cm}^{-1}\right)$ is the only transition which could be recorded (Fig. 9). The detection of vaporous water inside fuel cell channels is more difficult than measuring hydrogen or oxygen since at low temperatures the mole fraction of gaseous water just amounts a few percent of the gas mixture. For instance, the molar water content of water-saturated air is $4 \%$ at $25{ }^{\circ} \mathrm{C}$. For this reason, water vapor at room temperature could only be detected using strong laser powers (about $1 \mathrm{~W}$ ). Fig. 9 exhibits the evolution of the water vapor signal for three different temperatures.

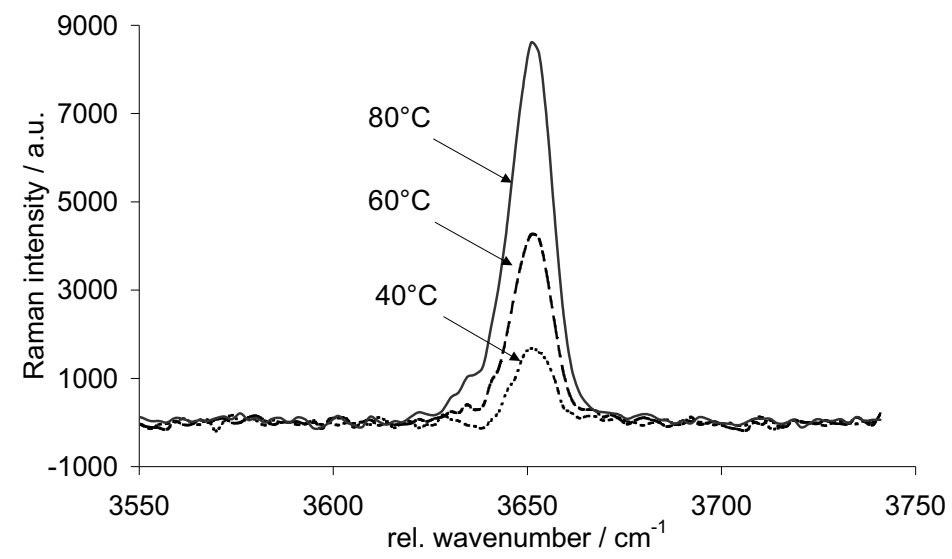

Fig. 9: The water vapor signal at different temperatures, $Q$ branch of the totally symmetric OH stretch mode, center of signal: $3652 \mathrm{~cm}^{-1}$; excitation wavelength: $477 \mathrm{~nm}$, laser power $1 \mathrm{~W}$, exposure time: $10 \mathrm{~min}$. 
Because of the high density of condensed media, liquid water inside the flow field channel can easily be detected. Since in condensed state intermolecular hydrogen bonding inhibit the independent motion of molecules, the Raman transitions become broad and lose their rotational substructure. Both $\mathrm{OH}$ stretch vibrations merge to one broad non-resolved transition with its centre at about $3400 \mathrm{~cm}^{-1}$.

\section{Multi-fiber Raman investigations}

This subsection presents some examples that show the time evolution of hydrogen and water vapor along the anode flow field.

At an advanced stage of aging, it is known that cell powers fluctuate considerably. The same behavior was found for the Raman signals of hydrogen at different measuring points. The Figure 10 relates the changes of hydrogen density to the global power of the cell as a function of time. Figure 10a represents the signal alterations at a measuring point next to the entrance of anode channel while Fig. $10 \mathrm{~b}$ shows the relations at the end of the serpentine channel.
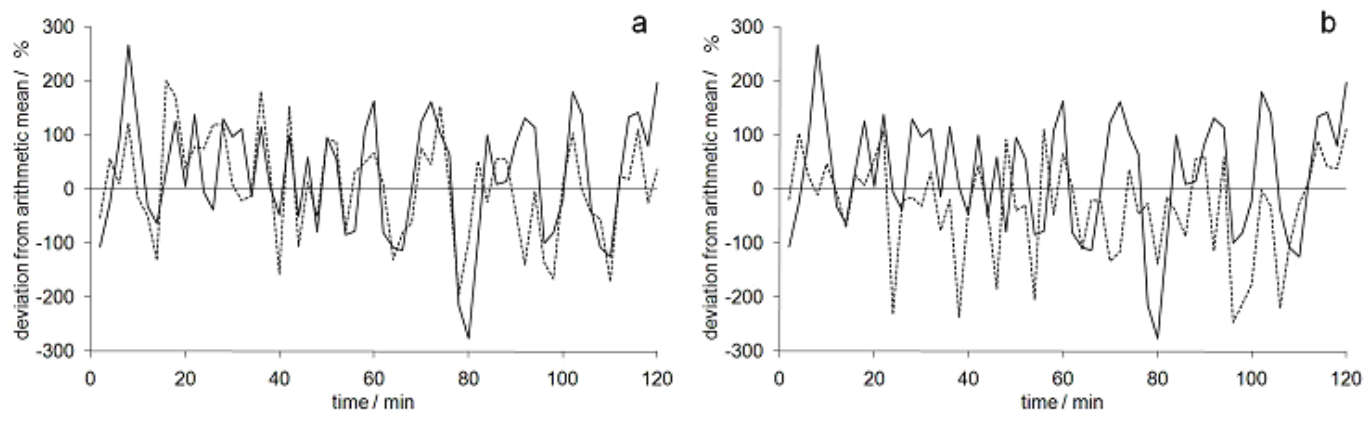

Fig. 10: Changes of the hydrogen density (dash line) and the global power (solid line) as a function of time at the entrance (a) and close to the end (b) of the anode serpentine (cell temperature: $55^{\circ} \mathrm{C}$ ). The hydrogen signals are inverted to better visualize the correspondence of both signals. The signal alterations are related to the mean values (el. power: $7.8 \mathrm{~W}$, Raman: peak area 63.5 in a. u.), respectively. The mean values are set to 0. The ordinate scale is related to the standard deviation (el. power: $0.8 \mathrm{~W}$, Raman: peak area 5.4 in a. u.) in percent.

Near to the entrance, the change of hydrogen density inversely follows the power changes in accordance with the chemical oxidation of hydrogen and the formation of electric current. This expected direct relation could solely be observed next to the entrance of the anode flow field. At the end of the anode serpentine a stronger incoherence between the changes of hydrogen signal and the power signal is evident. At the present state we attribute the fluctuations of hydrogen concentrations to the changing flow conditions within the flow field as a result of the high humidification of the anode. At the chosen temperature the humidification of the anode channel most likely induce condensation of water. The droplets inside the channel are then moved by the gas flow. In addition they can change their sizes. The cross sections of the channels are therefore continuously altered locally and in the course of time.

Fig. 11 presents the changes of water vapor signals at three different ports along the anode serpentine at $75{ }^{\circ} \mathrm{C}$ measured with Raman spectroscopy. The small value of water 
vapor means a low humidity next to the entrance. The low humidity is attributed to the high consumption of hydrogen by the electrochemical reaction since the transport of $\mathrm{H}^{+}$ ions apparently requires a considerable number of water molecules (electro-osmotic drag).

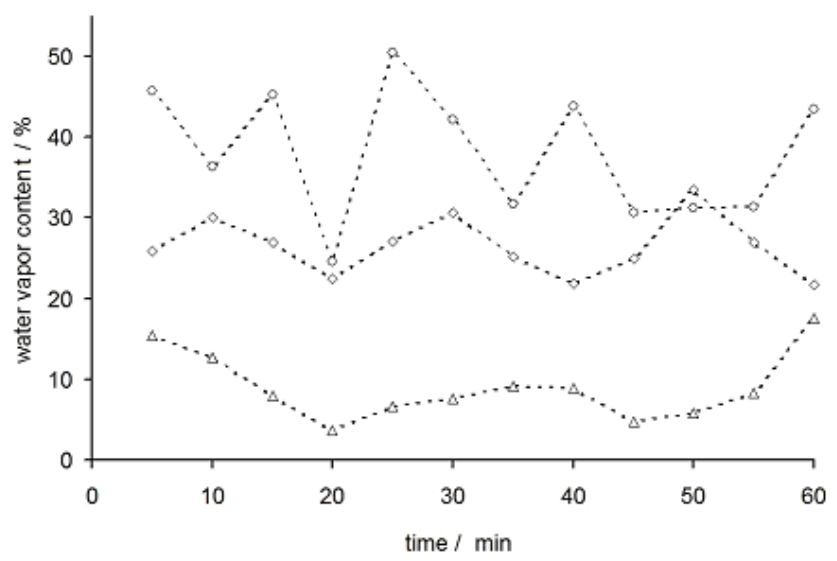

Fig. 11: Evolution of the water vapor signal as a function of time at three different points along the anode serpentine flow path; cell temperature: $75^{\circ} \mathrm{C}$; lower track: port next to the entrance, central track: port close to the center of the canal, upper track: close to the exit of the canal.

The graph in Figure 11 shows an increase of water vapor with increasing channel length. In addition the fluctuations of water vapor signals become stronger along the channel path. This is accompanied by a shortening of time periods in which changes of water vapor concentration take place. Condensation becomes more probable at the end of the serpentine flow since the water vapor content of the atmosphere of the channel increase along the flow path. This primarily leads to the observed local and temporal fluctuations of the water vapor concentration.

In Figure 12 measured hydrogen concentrations are compared to theoretically expected values for three representative measuring ports along the serpentine path. The ports are again located next to the entrance, in the center part of the flow field and at the end of the serpentine flow path.

The theoretically expected hydrogen concentration is calculated from the hydrogen consumption. That is derived from local current density measurements by applying Faraday's law. The theoretical hydrogen concentrations are scaled to the measured local humidity to obtain the gas composition in the flow field as shown in Figure 11. At the entrance the experimental hydrogen concentrations are smaller than expected. The gap between the calculated consumption and the measured data is attributed to diffusive losses. In the center of the serpentine the calculated data correspond well to the measured values. It is expected that the loss by diffusion becomes smaller since the partial pressure of hydrogen is already reduced. At the end of the flow path Because the measured values are higher compared to the expectation, however, due to the increased humidity and resulting condensation at the end of the anode serpentine, the data obtained in this region are difficult to interpret. A possibility is of course that the condensation of water leads to reduced activity in this region of the cell. 

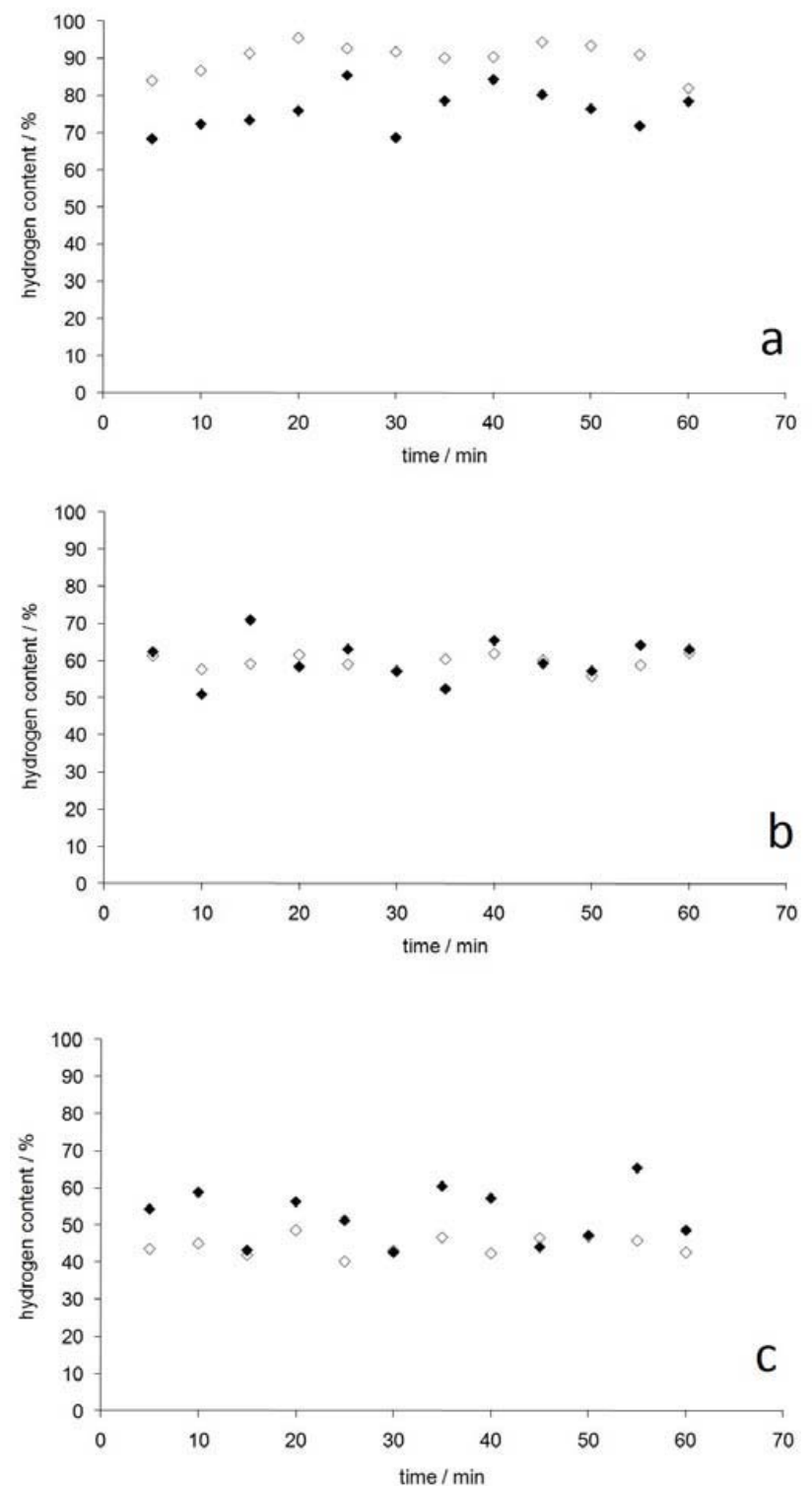

Fig. 12: Comparison of measured hydrogen content (filled dots) with calculated hydrogen content from local current density measurements corrected with local humidity (unfilled dots); the ordinary scale is referred to OCV operation (100\%); a: measuring port close to the entrance; $b$ : measuring port in the middle of the serpentine; $c$ : measuring port close to the end of the serpentine.

Summarizing, the spatially and temporally resolved Raman measurements along the anode serpentine have shown the dynamic changes in gas compositions while global power output is kept constant. Despite the natural interdependency between liquid water and water vapor, local changes of the water vapor content and liquid water do influence the local changes of hydrogen concentration considerably. In a next step, mutual relations 
between reactants can be evaluated in a coupled differential equation system in which the time derivatives of the species are considered as well as the local current densities. In addition to the current density distribution local impedance spectra will be concurrently detected.

\section{Conclusions}

Raman and EIS are developed as additional tools for locally resolved investigation of fuel cell operation. Both methods have potential to provide additional information about the local operating states. The time needed for measurements is for both methods in the range of few minutes, and allows investigating stationary or quasi-stationary states. Raman spectroscopy was successfully integrated into the cell at different position along the fuel flow path and the gas composition is analyzed. The detailed information and in particular deviations from expected gas compositions are used for an improved understanding of cell behavior which may be used in the future for optimization of cell as well as for improved control systems.

\section{Acknowledgement}

The authors would like to thank the German Federal Ministry of Education and Research (BMBF) for the financial support of the project "Online Diagnostik und Regelung für die PEFC".

\section{References}

1. T. E. Springer, T. A. Jr. Zawodzinski and S. Gottesfeld, Journal of the Electrochemical Society, 138, 2334 (1991).

2. R. Borup, J. Meyers, B. Pivovar, Y. S. Kim, R. Mukundan, N. Garland, D. Myers, M. Wilson, F. Garzon, D. Wood, P. Zelenay, K. More, K. Stroh, T. Zawodzinski, J. Boncella, J. E. McGrath, M. Inaba, K. Miyatake, M. Hori, K. Ota, Z. Ogumi, S. Miyata, A. Nishikata, Z. Siroma, Y. Uchimoto, K. Yasuda, K. Kimijima, and N. Iwashita, Chemical Reviews, 107, 3904 (2007).

3. J. Wu, X. Yuan, H. Wang, M. Blanco, J. Martin, and J. Zhang, International Journal of Hydrogen Energy, 33, 1735 (2008).

4. J. Wu, X. Ziyuan, H. Wang, M. Blanco, J. Martin, and J. Zhang, International Journal of Hydrogen Energy, 33, 1747 (2008).

5. M. Schulze, E. Gülzow, St. Schönbauer, T. Knöri, and R. Reissner, Journal of Power Sources, 173, 19 (2007).

6. X. Yuan, C. Song, H. Wang, and J. Zhang, Electrochemical Impedance Spectroscopy in PEM Fuel Cells (Springer London, London, 2010), p. 420.

7. A. Weber, Topics in Current Physics - Raman Spectroscopy of Gases and Liquids, $\mathrm{p}$. 318, Springer-Verlag, New York (1979).

8. D. Veirs and G. M. Rosenblatt, Journal of Molecular Spectroscopy, 121, 401 (1987).

9. A. Weber and E. A. Mcginnis, Journal of Molecular Spectroscopy, 4, 195 (1960).

10. S. A. Danichkin, A. A. Eliseev, T. N. Popova, O. V. Ravodina and V. V. Stenina, Zhurnal Prikladnoi Spectroskopii, 35, 581 (1981).

11. J. F. Ogilvie, The Vibrational and Rotational Spectrometry of Diatomic Molecules $\mathrm{p}$. 448, Academic Press, San Diego (1998).

12. W. F. Murphy, Molecular Physics, 36, 727 (1978). 